\title{
The long Non-coding RNA Orchestrator of Cancer Axis of Evil Insights into the Multiple Modes of Action of the H19 Gene
}

\author{
REVIEW
}

\section{Imad Matouk}

Department of Life Sciences, College of Science \& Technology, Al-Quds University, Jerusalem, Palestine

\begin{abstract}
A B S T R A C T
Increasing evidence has indicated that the non-coding RNA molecules play central roles in almost all biological processes and many pathological conditions including carcinogenesis. This review focuses on the pathological tumorigenic role of the first discovered long non-coding RNA gene called H19 and its pivotal contribution to the cancer axis of evil. H19 RNA utilizes a variety of mechanisms to perform its pathological function. Some key unanswered questions are presented by the end. Understanding the H19 RNA mechanisms of action will shed light into the class of long non-coding RNA which contains contains thousands of members mostly with unknown functionn and will help in delineating the pathological role played by at least some of them
\end{abstract}

Keywords: Long non-coding RNA, Competing endogenous RNA, Epigenetic regulation, microRNA sponge Epithelial to mesenchymal transition, Cancer stemness, Drug resistant, Exosome

After realizing that our DNA is a transcription machine, with a little protein coding potential, the past decade has witnessed an explosion in scientific researches reporting the identifications, characterizations and exploration of the modes of action of the noncoding RNA (ncRNA) genes. Currently, the ncRNA research field is one of the most popular fields in biological and medical sciences. An ncRNA is an RNA molecule that is not translated into a protein product and is classified according to its size into a long (more than 200 nucleotide) and a short (less than 200 nucleotide) ncRNA. The vast knowledge accumulated during the past decade was sufficient for the scientific community to announce the entry into the fascinating ncRNA era which was and still dominated by the

Correspondance: Department of Life Sciences, College of Science \& Technology, Jerusalem, Palestine.

E-mail: imatook@staff.alquds.edu

(C) copy rights 2021: All materials in this article is protected, permission requests should be addressed to Al-Quds University. www.alquds.edu
microRNAs (miRNA) researches. The miRNAs are small ncRNA mediating regulation of gene expression and their discovery has changed vastly the way we used to think about gene regulation.

The functions and mechanisms of action of the long non-coding RNAs (IncRNAs) are the least understood aspect of the ncRNA biology. Many thousands of IncRNAs are produced from our genome, yet relatively very few have well documented roles. This review will handle a gene called $\mathrm{H} 19$, which transcribes to an IncRNA.. Being the first imprinted IncRNA discovered, our knowledge on $\mathrm{H} 19$ is relatively high compared to the others. This is especially reflected by the steep increase in the scientific reports handling the $\mathrm{H} 19$ IncRNA functions and its modes of action that occurred in the past few years after decades of relative dormancy. $\mathrm{H} 19$ is expressed in the placenta and in the embryonic stage but shut down in most tissues after birth and re-expressed again in almost all cancer types.

The roles of $\mathrm{H} 19$ in cancer are diverse and 
touches almost every aspect of the tumorigenesic process. This review focuses into the wellestablished mechanistic roles of the H19 IncRNA in the epithelial to mesenchymal transition (EMT), cancer stem cells, and drug resistance, collectively called "cancer axis of evil" (Singh and Settleman, 2010), as they are responsible for the tumor metastasis and the failure of the chemotherapeutic drugs. This dictates indeed the cancer deadly signature. EMT is a reversible trans-differentiation process through which the non-motile epithelial cells are converted to motile mesenchymal cells, a step that is essential for tumor invasion and subsequent metastasis. Cancer stem cells are rare cancer cell population within the tumor that have stem-cell like properties and is greatly believed among the scientific community that these cells are responsible for fueling tumor growth, tumor heterogeneity, drug resistance and invasion. Some examples into other physiological and pathological roles for $\mathrm{H} 19$ are presented when needed for clarifications. It is astonishing how $\mathrm{H} 19$ can employs diverse mechanisms to perform its specific functions. An illustration of three (among others) well documented modes of action for $\mathrm{H} 19$ IncRNA is presented.

H19 InCRNA functions as a scavenger through sponging miRNAs.

One of the well-established modes of action through which the H19 InCRNA performs its function is by acting as a competing endogenous RNA (ceRNA) for the purpose of "sponging" appropriate candidate miRNAs. By this $\mathrm{H} 19$ relieves the sponged miRNA inhibitory effect on its downstream targets and thus acts as a scavenger.

One of the earliest reports showed a conserved role for $\mathrm{H} 19$ in modulation the major let-7 family availability by acting as a molecular sponge. Consequently, $\mathrm{H} 19$ affects expression of endogenous Let-7 targets including Dicer and Hmga2 (Kallen et al., 2013), a multifunctional proteins with broad activities. A double negative feedback loop between H19, let-7 miRNA and the pluripotency factor LIN28 has a critical role in the maintenance of breast cancer stem cell properties (Peng et al., 2018). Additional report indicates that oestrogen induction symmetric division in breast cancer stem-like cells is regulated by $\mathrm{H} 19$ through antagonizing Let-7c( Wang et al., 2019). We were the first to document that $\mathrm{H} 19$ is induced by hypoxic stress through the Hypoxia-inducible factor $1 \alpha$ (HIF1- $\alpha$ ) pathway (Matouk et al., 2010). An interesting recent report indicated that $\mathrm{H} 19$ is responsible for glycolysis and breast cancer stem cells (BCSC) maintenance. Mechanistically $\mathrm{H} 19$ acting as ceRNA sequesters miRNA let-7 miRNA to release HIF1- $\alpha$, leading to an increase in pyruvate dehydrogenase kinase 1 (PDK1) expression. PDK1 enhances BCSC properties and is correlated with poor overall survival (Peng et al., 2018).

We have provided several evidences for H19 central role in epithelial to mesenchymal transition (EMT) process (Matouk et al., 2014) and suggested that $\mathrm{H} 19$ can also act as an orchestrator for the EMT-MET processes (Matouk et al., 2016). Multiple reports have indicated that this can be performed at least in part through the sponging activity of H19 IncRNA. For instance, by derepressing let-7's suppression on its target HMGA2, H19 promotes EMT and metastasis in pancreatic cancer model ( $\mathrm{Ma}$ et al., 2013). Additionally, in colorectal cancer it was demonstrated that $\mathrm{H} 19$ sponges miR-138 and miR-200a that led to the de-repression of their endogenous targets Vimentin, ZEB1, and ZEB2, all are well established marker genes for mesenchymal cells (Liang et al., 2015) Furthermore it was shown that through differentially sponging $\mathrm{miR}-200 \mathrm{~b} / \mathrm{c}$ and let-7b, H19 mediates breast cancer cell plasticity during EMT and MET processes (Zhou et al., 2017). In glioma, H19 could compete with SOX4 via sponging miR-130a-3p and thus regulating EMT (Hu et al., 2018). H19 sponges miR-29b-3p and relieve the suppression for DNMT3B, which led to EMT and metastasis of bladder cancer (Lv et al., 2017).

By acting as ceRNA, several reports have indicated that $\mathrm{H} 19$ confer cancer chemoresistance in various models. H19 sponges miR-194-5p thus confers 5 -Fu resistance in colorectal cancer by promoting SIRT1-mediated autophagy (Wang et al., 2018). Additionally, H19 acts as a miRNA- 
106b-5p sponge and thus impairs the function of miRNA-106b-5p on its target gene, TDRG1. By this, $\mathrm{H} 19$ facilitate cell survival in cisplatin-based chemotherapeutic conditions in seminoma (Wei et al., 2018). Bortezomib resistance in multiple myeloma is also enhanced by $\mathrm{H} 19$ by acting as a miRNA sponge to inhibit the expression of miR29b-3p, enhance MCL-1 transcriptional translation and inhibit apoptosis (Pan et al., 2019). A recent study indicates that $\mathrm{H} 19$ confers resistance to gefitinib via miR-148b/dimethylarginine dimethylaminohydrolase-1 (DDAH1) axis in lung adenocarcinoma. Mechanistically, RNA H19 positively regulated DDAH1 expression via sponging miR-148b-3p (Huang et al., 2020).

\section{H19 is a precursor for miR-675-5p and miR-675-3p}

Discovered in 2007 miR-675 processed from the first and longest $\mathrm{H} 19$ exon (Cai and Cullen. 2007). MiR-675 was reported to target the "retinoplastoma gatekeeper of DNA replication" (Tsang et al., 2010) and the "p53 Guardian of the genome" (Zheng et al., 2019) both were among the best characterized and well established tumor suppressor genes. The inhibitory effect of miR675 on p53 could have a myriad consequences given hundreds of targets that p53 has the ability to modulate. We were the first to report that p53 suppress $\mathrm{H} 19$ induction upon hypoxic stress (Matouk et al,. 2010) and the upregulation of miR-675 in response to hypoxia (Matouk et al,. 2014). So the possibility of feedback loops between H19-miR-675-P53 by which miR-675b elevate the inhibitory effect of p53 on $\mathrm{H} 19$ upon hypoxic stress could be the case. Among other well-known tumor suppressor genes targeted by miR-675 is RUNX1 (Zhuang et al., 2014), and PTEN ( Lv et al., 2018) though the latter was not tested in the context of tumorigenicity. Thus it is not astonishing that at least part of the pathological role of $\mathrm{H} 19$ is mediated by miR675. MiR-675 targets a myriad of transcripts in a cellular-context-dependent manner involved in proliferation, apoptosis, EMT, invasion, migration, drug resistance, angiogenesis, and cancer stemness. Although some conflicting data have been reported in different research models, the prevailing view is that miR-675 is functioning as an onco-miR in most models. Physiologically, miR-675 is expressed exclusively in the placenta from the gestational time point when placental growth normally ceases. When lacking $\mathrm{H} 19$, the placentas continue to grow suggesting that the physiological role of $\mathrm{H} 19$ is to limit placental growth through its microRNA tool. Results indicate that miR-675 slows cell proliferation through at least in part targeting insulin like growth factor receptor 1 (Igf1r), the key receptor through which Igf2 signal to promote growth during fetal development (Keniry et al., 2012).

Additional physiological roles for miR-675 have been described in promoting skeletal muscle differentiation and regeneration ( Dey et al., 2014) and the regulation of intestinal epithelial barrier (Zou et al., 2016).

H19 IncRNA epigenetically modulate gene expression

Since H19's discovery in 1984, its locus has been used as a dogma to study epigenetic regulation of the imprinted genes where $\mathrm{H} 19$ also act in cis modulating and fine tuning the imprinting of other genes within the imprinted cluster where it resides. In this section the emerging role of $\mathrm{H} 19$ in epigenetic regulation of gene expression in Trans is handled which was uncovered in the past few years. H19 IncRNA interacts with transcription-repressors functioning epigenetically and guide them to specific loci. For example $\mathrm{H} 19$ binds the methylCpG-binding domain protein 1 (MBD1) and recruits it to some of its targets, by doing so $\mathrm{H} 19$ enables the maintaining of repressive H3K9me3 histone marks in their loci (Monnier et al., 2013). Enhancer of zeste homolog 2 (EZH2) is a critical component of Polycomb-Repressive Complex 2 (PRC2). EZH2 is responsible for generating histone $\mathrm{H} 3$ lysine 27 trimethylation, a modification that always correlates with transcriptionally repressed chromatin. Several reports uncover a "partnership" between $\mathrm{H}_{19}$ and EZH2. Even more dramatic are reports covering that $\mathrm{H} 19$ regulate $\mathrm{EZH} 2$ expression itself. EZH2 is regulated by $\mathrm{H} 19$, through sponging 
of miR-630 (Li et al., 2016). Additionally, and through sponging miR-130, it was reported that $\mathrm{H} 19$ regulate EZH2 expression (Hong et al. 2018). The functional outputs of $\mathrm{H} 19-\mathrm{EZH} 2$ association have been reported in a number of studies and using different models. Upregulated $\mathrm{H} 19$ enhances bladder cancer metastasis by associating with EZH2 and inhibiting E-cadherin expression. The authors showed that this association resulted in directly suppressing Ecadherin transcription and indirectly activating the Wnt signaling pathway (Luo et al., 2013). In tongue squamous cell carcinoma, it was shown that $\mathrm{H} 19$ promotes carcinoma progression through $\beta$-catenin / GSK3 $\beta / E M T$ signaling via association with EZH2 ( Zhang et al., 2017). Additionally, in esophageal cancer $\mathrm{H} 19$ facilitates EMT and metastasis through let-7c/STAT3/EZH2/ $\beta$ catenin axis (Chen et al., 2019). Furthermore, in glioblastoma cells $\mathrm{H} 19$ regulate NKD1 transcription via EZH2-induced H3K27 trimethylation of its promoter resulting in the repression of $\mathrm{Nkd1}-\mathrm{a}$ negative regulator of Wnt pathway (Fazi et al/.2018). In diabetic cardiomyopathy model, H19 inhibits autophagy by epigenetically silencing of DIRAS3. H19 knockdown could reduce EZH2 occupancy and $\mathrm{H} 3 \mathrm{~K} 27 \mathrm{me} 3$ binding in the promoter of DIRAS3 (Zhuo et al., 2017). Perhaps the most dramatic finding of the large scale (genome wide) epigenetic effect of H19 was reported by Zhou et al. (Zhou et al., 2015). In this report, it was shown that H19 binds to and inhibits S-adenosylhomocysteine hydrolase $(\mathrm{SAHH})$, the only mammalian enzyme capable of hydrolysing S-adenosylhomocysteine $(\mathrm{SAH})$. This enzyme is a potent feedback inhibitor of S-adenosylmethionine (SAM)-dependent methyltransferases that methylate nucleic acids, proteins and lipids. SAHH modulation by $\mathrm{H} 19$ thus exerts global effects by causing methylation changes at numerous gene loci genome-wide. This represents the first case in which $\mathrm{H} 19$ acts in trans to alter the epigenetic landscape genomewide.

The influence of H19 IncRNA extends beyond the cells transcribing it

With the discovery that RNA could be secreted outside the cells through exosomal vesicles, and cause phenotypic change in the cells receiving them, major changes about the local (cells transcribing it) phenotypic effect of these transcripts have been challenged with a very long believe that this function is only attributed to proteins. Cells communicated through RNA exosomes is relatively novel and could happen between normal cells, between cancer cells, and between normal and cancer cells. H19 IncRNA has functions far outside the cells transcribing it.Multiple reports indicate that exosomal H19 can induce drug resistant. For example exosomal H19 facilitated erlotinib and gefitinib resistance in non-small cell lung cancer (NSCLC) (Pan et al., 2020, Lei et al., 2020), H19 is delivered by exosomes to sensitive cells, leading to the dissemination of doxorubicin resistance in breast cancer (Wang et al.,2020). Carcinoma-associated fibroblasts promote the stemness and chemoresistance of colorectal carcinoma by transferring exosomal H19 ( Ren et al., 2018). Additional reports have indicated that exosomal $\mathrm{H} 19$ induce other phenotypes related to tumorigenesis including stemness, angiogenesis, cell invasion and migration and proliferation. Interestingly many of those phenotypes are induced in recipient cells with similar $\mathrm{H} 19$ scenarios of action to those described in the previous sections. In summation, it is increasingly clear that the H19 IncRNA is a major player and a corner stone of many facets of the tumorigenic processes. It's an ideal target for therapeutic intervention. It uses diverse tools embedded in its primary RNA sequences to act as a sponger or a producer of microRNA (Figure 1).

Globally, H19 modify gene expression through epigenetic regulation genome wide. Despite this impressive progress made in the past decade, still we have many points need to be addressed for better understanding.We have highlighted the importance of $\mathrm{H} 19$ IncRNA during many stages of tumorigenesis. We are aware that the vast scenarios presented above are collected from different models and situations. So what dictates the mode of action? For instance, E-cadherin is suppressed by $\mathrm{H} 19$ by at least three modes of action as a sponger and a producer of microRNA, and also through epigenetic regulation. 


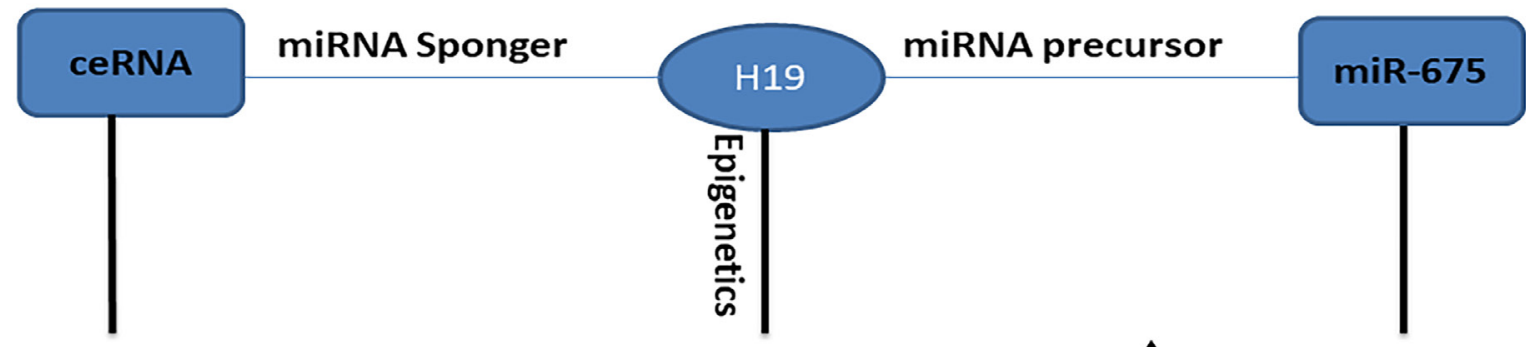

let-7, miR-138, miR-200 a, miR200b/c, miR-130a-3p, miR 29b-3p
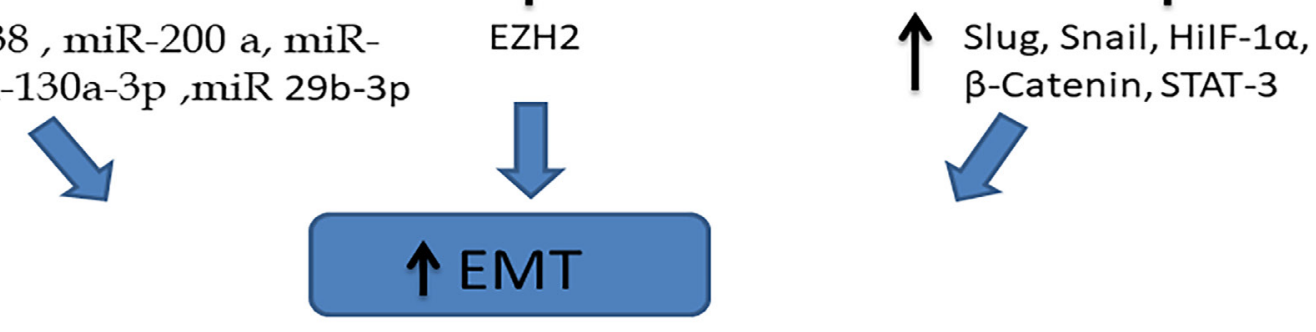

$\mathrm{EZH} 2$

$\beta$-Catenin, STAT-3

Figure 3: H19 IncRNA induces EMT via multiple mechanisms of action

By acting as competing endogenous RNA, miRNA precursor, and epigenetic regulator, $\mathrm{H} 19$ IncRNA induces EMT. Shown are some of the validated targets of H19 and its miRNA miR-675.

Are these scenarios happening concurrently in the same cancer cells to assure E-cadherin suppression or each scenario is happening in a tissue-specific manner? What triggers the release of $\mathrm{H} 19$ through the exosomes? Do H19 and miR-675 have different regulatory sequences, taking into consideration that they are both upregulated in many cancer types and it seems that miR-675 is not produced an the expense of H19. Why are still some reports arguing for a tumor suppressor function of $\mathrm{H} 19$ and its miR675- Is H19 gene locus playing a dual role? If yes what triggers each role. Another question that needs to be addressed is whether H19 IncRNA is also acting as an antisense transcript given that its locus also transcribed in antisense direction.

Certainly, understanding the different mode of actions performed by $\mathrm{H} 19$ IncRNA will foster our thoughts towards delineating the possible roles and the mechanism of action of so many IncRNA with unknown function. It is not odd to speculate that the mechanistic mode of action of $\mathrm{H} 19$ is shared by other IncRNAs.

\section{Competing interest}

The author declares no competing interest to disclose.

\section{References}

Cai, X. and Cullen, B.R., 2007. The imprinted H19 noncoding RNA is a primary microRNA precursor. Rna, 13(3), pp.313-316.

Chen, M.J., Deng, J., Chen, C., Hu, W., Yuan, Y.C. and Xia, Z.K., 2019. LncRNA H19 promotes epithelial mesenchymal transition and metastasis of esophageal cancer via STAT3/EZH2 axis. The International Journal of Biochemistry \& Cell Biology, 113, pp.27-36.

Dey, B.K., Pfeifer, K. and Dutta, A., 2014. The H19 long noncoding RNA gives rise to microRNAs miR675-3p and miR-675-5p to promote skeletal muscle differentiation and regeneration. Genes \& development, 28(5), pp.491-501.

Fazi, B., Garbo, S., Toschi, N., Mangiola, A., Lombari, M., Sicari, D., Battistelli, C., Galardi, S., Michienzi, A., Trevisi, G. and Harari-Steinfeld, R., 2018. The IncRNA H19 positively affects the tumorigenic properties of glioblastoma cells and contributes to NKD1 repression through the recruitment of EZH2 on its promoter. Oncotarget, 9(21), p.15512.

Hong, Y., He, H., Sui, W., Zhang, J., Zhang, S. and Yang, D., 2018. [Corrigendum] Long non coding RNA $\mathrm{H} 19$ promotes cell proliferation and invasion by acting as a ceRNA of miR 138 and releasing EZH2 in oral squamous cell carcinoma. International journal of oncology, 53(2), pp.915-915.

Hu, Q., Yin, J., Zeng, A., Jin, X., Zhang, Z., Yan, W. and 
You, Y., 2018. H19 functions as a competing endogenous RNA to regulate EMT by sponging miR-130a-3p in glioma. Cellular Physiology and Biochemistry, 50(1), pp.233-245.

Huang, Z., Ma, Y., Zhang, P., Si, J., Xiong, Y. and Yang, Y., 2020. Long non-coding RNA H19 confers resistance to gefitinib via miR-148b-3p/DDAH1 axis in lung adenocarcinoma. Anti-Cancer Drugs, 31(1), pp.44-54.

Kallen, A.N., Zhou, X.B., Xu, J., Qiao, C., Ma, J., Yan, L., Lu, L., Liu, C., Yi, J.S., Zhang, H. and Min, W., 2013. The imprinted H19 IncRNA antagonizes let-7 microRNAs. Molecular cell, 52(1), pp.101-112.

Keniry, A., Oxley, D., Monnier, P., Kyba, M., Dandolo, L., Smits, G. and Reik, W., 2012. The H19 lincRNA is a developmental reservoir of miR-675 that suppresses growth and Igf1r. Nature cell biology, 14(7), pp.659-665.

Lei, Y., Guo, W., Chen, B., Chen, L., Gong, J. and Li, W., 2018. Tumor released IncRNA H19 promotes gefitinib resistance via packaging into exosomes in non small cell lung cancer. Oncology Reports, 40(6), pp.3438-3446.

Li, X., Lin, Y., Yang, X., Wu, X. and He, X., 2016. Long noncoding RNA H19 regulates EZH2 expression by interacting with miR-630 and promotes cell invasion in nasopharyngeal carcinoma. Biochemical and biophysical research communications, 473(4), pp.913-919.

Liang, W.C., Fu, W.M., Wong, C.W., Wang, Y., Wang, W.M., Hu, G.X., Zhang, L., Xiao, L.J., Wan, D.C.C., Zhang, J.F. and Waye, M.M.Y., 2015. The IncRNA $\mathrm{H} 19$ promotes epithelial to mesenchymal transition by functioning as miRNA sponges in colorectal cancer. Oncotarget, 6(26), p.22513.

Luo, M., Li, Z., Wang, W., Zeng, Y., Liu, Z. and Qiu, J., 2013. Long non-coding RNA H19 increases bladder cancer metastasis by associating with $\mathrm{EZH} 2$ and inhibiting E-cadherin expression. Cancer letters, 333(2), pp.213-221.

Lv, J., Wang, L., Zhang, J., Lin, R., Wang, L., Sun, W., Wu, H. and Xin, S., 2018. Long noncoding RNA H19-derived miR-675 aggravates restenosis by targeting PTEN. Biochemical and biophysical research communications, 497(4), pp.1154-1161.

Lv, M., Zhong, Z., Huang, M., Tian, Q., Jiang, R. and Chen, J., 2017. IncRNA H19 regulates epithelialmesenchymal transition and metastasis of bladder cancer by miR-29b-3p as competing endogenous RNA. Biochimica et Biophysica Acta (BBA)-Molecular Cell Research, 1864(10), pp.1887-1899.

Ma, C., Nong, K., Zhu, H., Wang, W., Huang, X., Yuan, Z. and $\mathrm{Ai}, \mathrm{K} ., 2014$. H19 promotes pancreatic cancer metastasis by derepressing let-7's suppression on its target HMGA2-mediated EMT. Tumor Biology, 35(9), pp.9163-9169.

Matouk, I.J., Mezan, S., Mizrahi, A., Ohana, P., Abu-lail, R., Fellig, Y., deGroot, N., Galun, E. and Hochberg, A., 2010. The oncofetal H19 RNA connection: hypoxia, p53 and cancer. Biochimica et Biophysica Acta (BBA)-Molecular Cell Research, 1803(4), pp.443-451.

Matouk, I.J., Raveh, E., Abu-lail, R., Mezan, S., Gilon, M., Gershtain, E., Birman, T., Gallula, J., Schneider, T., Barkali, M. and Richler, C., 2014. Oncofetal H19 RNA promotes tumor metastasis. Biochimica et Biophysica Acta (BBA)-Molecular Cell Research, 1843(7), pp.1414-1426.

Matouk, I.J., Halle, D., Raveh, E., Gilon, M., Sorin, V. and Hochberg, A., 2016. The role of the oncofetal H19 IncRNA in tumor metastasis: orchestrating the EMT-MET decision. Oncotarget, 7(4), p.3748.

Monnier, P., Martinet, C., Pontis, J., Stancheva, I., Ait-Si-Ali, S. and Dandolo, L., 2013. H19 IncRNA controls gene expression of the Imprinted Gene Network by recruiting MBD1. Proceedings of the National Academy of Sciences, 110(51), pp.20693-20698.

Pan, R. and Zhou, H., 2020. Exosomal Transfer of IncRNA H19 Promotes Erlotinib Resistance in Non-Small Cell Lung Cancer via miR-615-3p/ ATG7 Axis. Cancer Management and Research, 12, pp.4283-4297.

Pan, Y., Zhang, Y., Liu, W., Huang, Y., Shen, X., Jing, R., Pu, J., Wang, X., Ju, S., Cong, H. and Chen, H., 2019. LncRNA H19 overexpression induces bortezomib resistance in multiple myeloma by targeting MCL-1 via miR-29b-3p. Cell death \& disease, 10(2), pp.1-14.

Peng, F., Li, T.T., Wang, K.L., Xiao, G.Q., Wang, J.H., Zhao, H.D., Kang, Z.J., Fan, W.J., Zhu, L.L., Li, M. and Cui, B., 2018. H19/let-7/LIN28 reciprocal negative regulatory circuit promotes breast cancer stem cell maintenance. Cell death \& disease, 8(1), pp.e2569-e2569.

Peng, F., Wang, J.H., Fan, W.J., Meng, Y.T., Li, M.M., Li, T.T., Cui, B., Wang, H.F., Zhao, Y., An, F. and Guo, T., 2018. Glycolysis gatekeeper PDK1 reprograms breast cancer stem cells under hypoxia. Oncogene, 37(8), pp.1062-1074.

Ren, J., Ding, L., Zhang, D., Shi, G., Xu, Q., Shen, S., Wang, Y., Wang, T. and Hou, Y., 2018. Carcinomaassociated fibroblasts promote the stemness and chemoresistance of colorectal cancer by transferring exosomal IncRNA H19. Theranostics, 
8(14), p.3932.

Singh, A., \& Settleman, J. E. M. T. 2010. EMT, cancer stem cells and drug resistance: an emerging axis of evil in the war on cancer. Oncogene, 29(34), 4741-4751.

Tsang, W.P., Ng, E.K., Ng, S.S., Jin, H., Yu, J., Sung, J.J. and Kwok, T.T., 2010. Oncofetal H19-derived miR675 regulates tumor suppressor RB in human colorectal cancer. Carcinogenesis, 31(3), pp.350358.

Wang, M., Han, D., Yuan, Z., Hu, H., Zhao, Z., Yang, R., Jin, Y., Zou, C., Chen, Y., Wang, G. and Gao, X., 2018. Long non-coding RNA H19 confers 5-Fu resistance in colorectal cancer by promoting SIRT1-mediated autophagy. Cell death \& disease, 9(12), pp.1-14.

Wang, M., Li, Y., Xiao, G.D., Zheng, X.Q., Wang, J.C., Xu, C.W., Qin, S., Ren, H., Tang, S.C. and Sun, X., 2019. H19 regulation of oestrogen induction of symmetric division is achieved by antagonizing Let-7c in breast cancer stem-like cells. Cell proliferation, 52(1), p.e12534.

Wang, X., Pei, X., Guo, G., Qian, X., Dou, D., Zhang, Z., Xu, X. and Duan, X., 2020. Exosome-mediated transfer of long noncoding RNA H19 induces doxorubicin resistance in breast cancer. Journal of Cellular Physiology.

Wei, J., Gan, Y., Peng, D., Jiang, X., Kitazawa, R., Xiang, Y., Dai, Y., Tang, Y. and Yang, J., 2018. Long noncoding RNA H19 promotes TDRG1 expression and cisplatin resistance by sequestering miRNA-106b-5p in seminoma. Cancer medicine, 7(12), pp.6247-6257.

Zhang, D.M., Lin, Z.Y., Yang, Z.H., Wang, Y.Y., Wan, D., Zhong, J.L., Zhuang, P.L., Huang, Z.Q., Zhou, B. and Chen, W.L., 2017. IncRNA H19 promotes tongue squamous cell carcinoma progression through $\beta$-catenin/GSK3 $\beta / E M T$ signaling via association with EZH2. American journal of translational research, 9(7), p.3474.
Zhuang, M., Gao, W., Xu, J., Wang, P. and Shu, Y., 2014. The long non-coding RNA H19-derived miR-675 modulates human gastric cancer cell proliferation by targeting tumor suppressor RUNX1. Biochemical and biophysical research communications, 448(3), pp.315-322.

Zheng, Z.H., Wu, D.M., Fan, S.H., Zhang, Z.F., Chen, G.Q. and Lu, J., 2019. Upregulation of miR-675-5p induced by IncRNA H19 was associated with tumor progression and development by targeting tumor suppressor p53 in nonsmall cell lung cancer. Journal of Cellular Biochemistry, 120(11), pp.18724-18735.

Zhou, J., Yang, L., Zhong, T., Mueller, M., Men, Y., Zhang, N., Xie, J., Giang, K., Chung, H., Sun, X. and Lu, L., 2015. H19 IncRNA alters DNA methylation genome wide by regulating S-adenosylhomocysteine hydrolase. Nature communications, 6(1), pp.1-13.

Zhou, W., Ye, X.L., Xu, J., Cao, M.G., Fang, Z.Y., Li, L.Y., Guan, G.H., Liu, Q., Qian, Y.H. and Xie, D., 2017. The IncRNA H19 mediates breast cancer cell plasticity during EMT and MET plasticity by differentially sponging miR-200b/c and let-7b. Science signaling, 10(483).

Zhuo, C., Jiang, R., Lin, X. and Shao, M., 2017. LncRNA H19 inhibits autophagy by epigenetically silencing of DIRAS3 in diabetic cardiomyopathy. Oncotarget, 8(1), p.1429.

Zou, T., Jaladanki, S.K., Liu, L., Xiao, L., Chung, H.K., Wang, J.Y., Xu, Y., Gorospe, M. and Wang, J.Y., 2016. H19 long noncoding RNA regulates intestinal epithelial barrier function via microRNA 675 by interacting with RNA-binding protein HuR. Molecular and cellular biology, 36(9), pp.1332-1341. 